\title{
Comportamento elétrico e magnético da cerâmica Ni-Zn
}

\author{
Valesca Donizeti de Oliveira ${ }^{1 *}$ (10) \\ Claudiney de Sales Pereira Mendonça ${ }^{2}$ \\ Manuel Ribeiro da Silva ${ }^{1}$ \\ Rero Marques Rubinger ${ }^{2}$ \\ Adhimar Flávio Oliveira ${ }^{2}$ \\ Vander Alkmin dos Santos Ribeiro ${ }^{3}$
}

\section{Resumo}

As ferritas receberam um renovado interesse tecnológico e científico nos últimos anos, devido ao seu baixo custo de produção e à diversidade de aplicações. As ferritas de níquel-zinco são bem conhecidas como materiais magnéticos moles com boas propriedades, tais como alta resistividade elétrica, baixa perda de corrente parasita em altas frequências e propriedades de aquecimento por indução em um campo magnético alternado (a.c.). Elas são amplamente utilizadas em diversos produtos industriais, como transformadores de potência, micro-ondas e equipamentos de telecomunicações. Amostras de ferritas de níquel-zinco $\left(\mathrm{Ni}_{\mathrm{x}} \mathrm{Zn}_{1-\mathrm{x}} \mathrm{Fe}_{2} \mathrm{O}_{4}, \operatorname{com} \mathrm{x}=0,00,0,25,0,50,0,75,1,00\right)$ foram sinterizadas a $1250{ }^{\circ} \mathrm{C}$ por $20 \mathrm{~h}$ usando o método de reação de estado-sólido. A estrutura cúbica de espinélio foi a microestrutura encontrada na série de ferritas Ni-Zn. A estrutura do sistema espinélio misto foi investigada com difração de raios X. As propriedades magnéticas foram caracterizadas com um magnetômetro de amostra vibrante, pelo qual foi obtida a curva de magnetização em função do campo magnético aplicado. A condutividade elétrica DC das pastilhas cilíndricas foi caracterizada com variação da temperatura de $30^{\circ} \mathrm{C}$ a $550{ }^{\circ} \mathrm{C}$. Os resultados da caracterização elétrica mostram que as ferritas de níquel-zinco produzidas apresentam características semicondutoras.

Palavras-chave: Ferrita níquel-zinco; Magnetização de saturação; Espinélio.

\section{Electric and magnetic behavior of Ni-Zn ceramic}

\begin{abstract}
Ferrites have received a renewed technological and scientific interest in recent years, due to their low cost of production and the diversity of applications. Nickel-zinc ferrites are well known as soft magnetic materials with good properties, such as high electrical resistivity, low loss of eddy current at high frequencies and induction heating properties in an alternating magnetic field (a.c.). They are widely used in various industrial products, such as power transformers, microwaves and telecommunications equipment. Samples of nickel-zinc ferrites $\left(\mathrm{Ni}_{\mathrm{x}} \mathrm{Zn}_{1-\mathrm{x}} \mathrm{Fe}_{2} \mathrm{O}_{4}\right.$, with $\mathrm{x}=0.00,0.25,0.50$, $0.75,1.00$ ) were sintered at $1250{ }^{\circ} \mathrm{C}$ for $20 \mathrm{~h}$ using the state reaction method -solid. The spinel cubic structure was the microstructure found in the Ni-Zn ferrite series. The structure of the mixed spinel system was investigated with X-ray diffraction. The magnetic properties were characterized with a vibrating sample magnetometer, by which the magnetization curve was obtained as a function of the applied magnetic field. The DC electrical conductivity of cylindrical pallets was characterized with temperature variation from $30{ }^{\circ} \mathrm{C}$ to $550{ }^{\circ} \mathrm{C}$. The results of the electrical characterization show that the nickel-zinc ferrites produced have semiconductor characteristics.
\end{abstract}

Keywords: Nickel-zinc ferrite; Magnetization saturation; Spinel.

${ }^{I}$ Instituto de Engenharia Mecânica, Universidade Federal de Itajubá - UNIFEI, Itajubá, MG, Brasil.

${ }^{2}$ Instituto de Física e Química, Universidade Federal de Itajubá - UNIFEI, Itajubá, MG, Brasil.

${ }^{3}$ Centro Universitário de Itajubá - FEPI, Itajubá, $M G$, Brasil.

*Autor correspondente: valescaoliveira@unifei.edu.br

2176-1523 (C) 2021. Oliveira et al. Publicado pela ABM. Este é um artigo publicado em acesso aberto (Open Access) sob a licença Creative Commons Attribution, que permite uso, distribuição e reprodução em qualquer meio, sem restrições desde que o trabalho original seja corretamente citado. 


\section{Introdução}

Um dos materiais ferrimagnéticos mais importantes são óxidos duplos de ferro e outro elemento metálico que são denominados de ferrita. As ferritas têm sido amplamente utilizadas em várias aplicações tecnológicas incluindo telecomunicações, fontes de alimentação comutadas, motores de corrente contínua/alternada, memórias digitais, sensores de gases, transporte de fármacos, indutores de multicamadas e transformadores [1,2].

As ferritas de níquel, $\mathrm{NiFe}_{2} \mathrm{O}_{4}$ têm várias aplicações tecnológicas tais como: catalisadores heterogêneos [3], agente de contraste para ressonância magnética [4], e sistema de alta frequência [5]. As ferritas de zinco têm algumas aplicações como: catálise e fotocatálise [6] e apresentam uma alta permeabilidade, tornando-as úteis para a fabricação de equipamentos elétricos [7].

A preferência de elementos não magnéticos em ferritas de estrutura cristalina de espinélio alteram suas propriedades magnéticas e elétricas. Estudos revelaram informações úteis sobre a natureza da interação de troca, a direção da magnetização e a distribuição de cátions. Nanopartículas magnéticas estão se tornando cada vez mais interessantes em várias áreas $[8,9]$.

As amostras apresentadas neste manuscrito têm uma estrutura cristalina cúbica de espinélio, cuja célula unitária tem a fórmula estrutural genérica $\left[\mathrm{M}_{1-\delta}{ }_{-} \mathrm{Fe}_{1-\delta}\right]^{\mathrm{A}}\left[\mathrm{M}_{\delta} \mathrm{Fe}_{(2+\delta)}\right]^{\mathrm{B}} \mathrm{O}_{4}$, em que $\delta$ é um índice estequiométrico que atua como o parâmetro de inversão com $\delta=0$ para espinélios normais e $\delta=1$ para os espinélios inversos [10]. Os valores entre colchetes representam a ocupação média dos sítios tetraédricos (A) e octaédricos (B). Caracteriza-se como espinélio normal o arranjo de cátions bivalentes nos oito interstícios tetraédricos e os cátions trivalentes nos dezesseis interstícios octaédricos. Os espinélios inversos são aqueles que apresentam os cátions bivalentes nas oito posições tetraédricas, enquanto os cátions trivalentes ocupam igualmente as oito posições intersticiais tetraédricas e octaédricas. Os espinélios mistos ou intermediários assumem a seguinte condição $0<\delta<1$ [11].

A formação de óxidos complexos com a estrutura espinélio usando a reação de estado-sólido entre óxidos simples ocorre lentamente e requer uma exposição prolongada a temperaturas consideradas elevadas [12]. Altas temperaturas por longos períodos são necessárias para que os íons tenham energia térmica suficiente para ocasionalmente vibrar ou saltar de um local para uma vacância adjacente ou intersticial [13]. Verificou-se que a temperatura de sinterização tem forte efeito sobre a microestrutura e como tal pode afetar as propriedades magnéticas [14].

Os íons de níquel e zinco têm forte preferência por sítios tetraédricos e octaédricos, respectivamente. As ferritas mistas de Ni-Zn apresentam diferentes composições estequiométricas dos elementos envolvidos o que resulta numa redistribuição dos íons metálicos nos sítios tetraédricos e octaédricos que podem modificar as propriedades da ferrita [15]. Os sítios tetraédricos são menores que os octaédricos em ferritas e em outros óxidos tipo espinélio. Isto se deve ao fato que sítios tetraédricos são pequenos demais para conter um íon metálico quando se considera íons como esferas sólidas.

O modelo Potencial de Barreira nos Contornos de Grão deve ser aplicável em materiais que contêm contornos de grãos com alta resistência elétrica. A formação da camada resistiva presente nas regiões de contornos de grão, que envolvem os grãos semicondutores, resulta na criação de barreiras de potencial que impedem a passagem de elétrons [16]. O surgimento da barreira potencial impede o movimento de transportadores, e por consequência, reduz a mobilidade dos portadores de carga. Portanto, o contorno de grão afeta fortemente a condutividade elétrica dos sólidos policristalinos [17].

A resistividade elétrica, $\rho$, é uma propriedade elétrica dos materiais. No mecanismo de condução elétrica pelo contorno de grão, a variável resistividade é uma função exponencial da temperatura, bem como outras informações correlatas. Neste método, a resistividade é dada pela Equação 1 [18].

$$
\rho=\left(\frac{2 \pi m^{*} K_{B} T}{(L / 2) e^{2} n}\right) \exp \left(\frac{E_{b}}{K_{B} T}\right)
$$

Designa-se por $e$ a carga elementar do elétron $\left(1,916.10^{-19} \mathrm{C}\right), K_{B}$ a constante de Boltzmann $\left(8,617.10^{-5} \mathrm{eV} / \mathrm{K}\right), n$ a concentração de elétrons nos cristalitos, $L / 2$ o tamanho médio dos cristalitos, $m^{*}$ a massa efetiva dos portadores de carga e $E_{B}$ a altura da barreira de energia no contorno do grão [18].

$$
E_{B}=\frac{(L / 2)^{2} e^{2} N_{D}}{8 \varepsilon}
$$

Designa-se por $\varepsilon$ permissividade a baixa frequência, $N_{D}$ a concentração de estados doadores. O comprimento médio do cristalito, $L / 2$, é determinado pela equação de Scherrer através da técnica de difração de raios X. A altura da barreira de potencial é altamente influenciada pela condutividade do grão.

Neste trabalho, apresenta-se as etapas de preparação das amostras via reação do estado sólido de ferritas mistas de zinco-níquel, a caracterização elétrica em corrente contínua e alternada. A caracterização magnética realizada via magnetômetro de amostra vibrante em temperatura ambiente e a caracterização microestrutural é feita por difração de raios X. De acordo com as caracterizações as amostras apresentam caráter semicondutor e seu comportamento elétrico permitiu a modelagem por potencial de barreiras nos contornos dos cristalitos, obtendo propriedades nunca antes exploradas neste material.

\section{Procedimento experimental}

Os óxidos de ferro e zinco de alta pureza foram cuidadosamente separados e pesados para então serem misturados com o óxido de magnésio para obter a série de ferrita 
$\mathrm{Ni}_{\mathrm{x}} \mathrm{Zn}_{1-\mathrm{x}} \mathrm{Fe}_{2} \mathrm{O}_{4}(\mathrm{NZFO})$. Os compostos químicos utilizados para a obtenção das amostras possuem pureza analítica superior $99,00 \%$ da Sigma Aldrich. A mistura homogeneizada dos pós foi compactada em um molde metálico de diâmetro de $8 \mathrm{~mm}$ (Figura 1). com uma pressão uniaxial de $400 \mathrm{MPa}$. Foram produzidas 2 gramas para cada estequiometria em quatro pastilhas cilíndricas de aproximadamente 0,5 grama.

Depois disso, as amostras de $\mathrm{Ni}_{x} \mathrm{Zn}_{1-\mathrm{x}} \mathrm{Fe}_{2} \mathrm{O}_{4}$ foram sinterizadas a $1250{ }^{\circ} \mathrm{C}$ por $20 \mathrm{~h}$. As fases cristalinas foram identificadas por difração de raios $\mathrm{X}$ utilizando um difratômetro Panalytical X'pert, com radiação $\mathrm{Cu}-\mathrm{k}_{\alpha}$ $1,5405 \mathrm{~nm}$ com intervalo angular $2 \theta$ de $15^{\circ}$ a $80^{\circ}$, com um passo de $0,02^{\circ}$ segundo. A condutividade detelétrica foi realizada através de uma fonte meter Keithley $2400 \mathrm{com}$ variação da temperatura entre $30^{\circ} \mathrm{C} \mathrm{e} 550^{\circ} \mathrm{C}$ com uma taxa de aquecimento de $3^{\circ} \mathrm{C}$ por minuto. A medida da temperatura no forno foi realizada através de um multímetro Keithey 2100. Todos os dados foram coletados através de um software em LabView. A caracterização magnética foi realizada com o magnetômetro de amostra vibrante Lakeshore com um campo magnético aplicado de $\pm 14 \mathrm{kG}$.

\section{Resultado e discussão}

A Figura 2 representa os difratogramas de raios $\mathrm{X}$ das amostras da série de ferrita NZFO. Pode-se observar que os picos de difração correspondem ao espinélio padrão (ficha JCPDS - 00-052-0277). Os padrões de difração confirmaram uma estrutura cúbica que não apresenta picos extras (o que poderia indicar outras fases). Os picos foram indexados em (111), (022), (133), (222), (224), (333) e (044) para os planos de uma célula unitária cúbica. $O$ deslocamento dos picos para a esquerda e para a direita pode ser explicado pelo aumento e diminuição do parâmetro de rede, respectivamente. Pode ser visto que o deslocamento do pico de maior intensidade para a esquerda indica o aumento do parâmetro de rede.

A Figura 3 mostra a curva de magnetização à temperatura ambiente de todas as amostras. Podemos observar que a saturação é atingida em campos relativamente baixos (14kG).

A curva de magnetização permite determinar parâmetros magnéticos como o campo coercitivo $(\mathrm{Hc})$, magnetização remanente (Mr) e magnetização de saturação (Ms). As propriedades magnéticas das ferritas níquel-zinco obtidas a partir das curvas de magnetização são mostradas na Tabela 1.

A amostra $\mathrm{Ni}_{0,50} \mathrm{Zn}_{0,50} \mathrm{Fe}_{2} \mathrm{O}_{4}$ apresentou maior valor de magnetização de saturação, enquanto a amostra $\mathrm{Ni}_{0,75} \mathrm{Zn}_{0,25} \mathrm{Fe}_{2} \mathrm{O}_{4}$ apresentou maiores valores de campo coercitivo e magnetização remanescente.

A variação da magnetização de saturação pode ser correlacionada à distribuição de cátions devido à interação de troca de íons tetraédricos (A) e octaédricos (B). A variação observada da magnetização com a concentração de $Z n$ pode ser explicada usando a aproximação de campo molecular e três modelos de sub-redes [19].

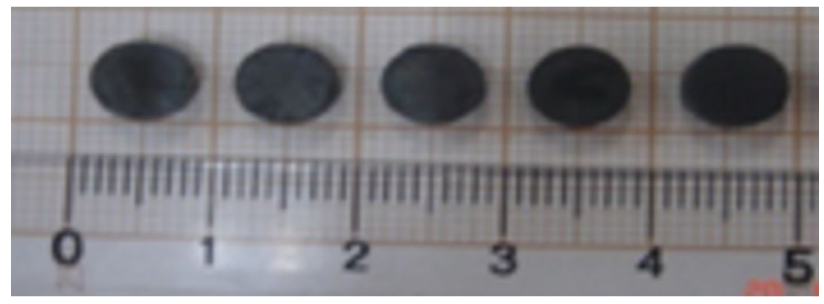

Figura 1. Geometria das pastilhas das ferritas níquel-zinco.

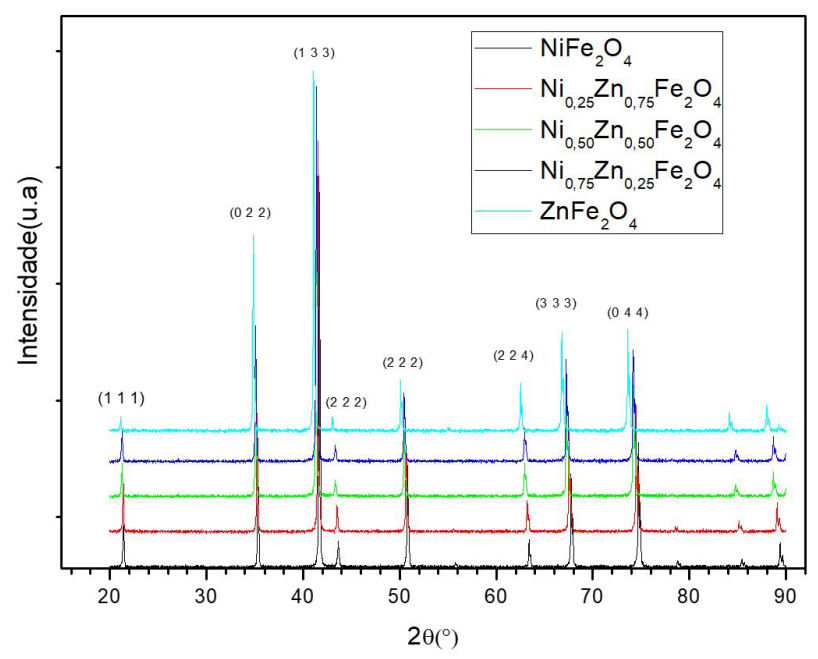

Figura 2. Difratograma de raios $\mathrm{X}$ das ferritas níquel-zinco.

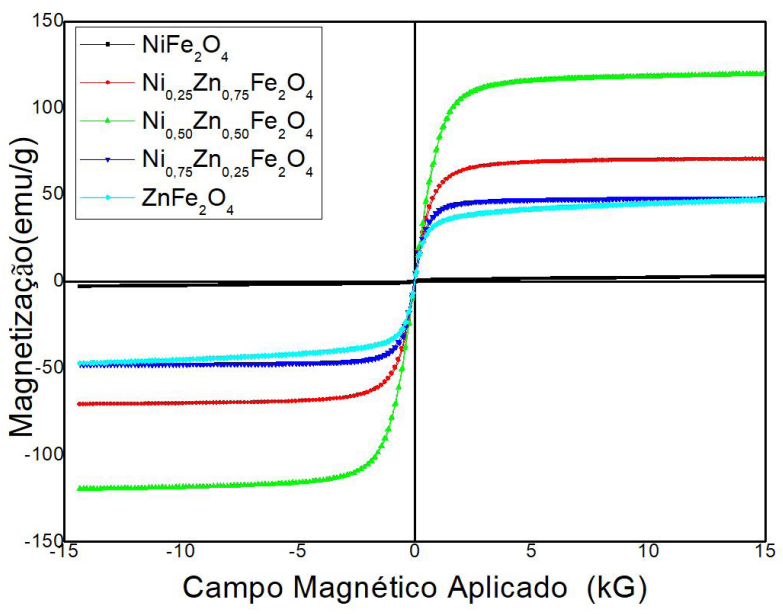

Figura 3. Curva de magnetização da ferrita níquel- zinco.

Tabela 1. Resultado de magnetização da curva de magnetização $\mathrm{Ni}_{x} \mathrm{Zn}_{1-x} \mathrm{Fe}_{2} \mathrm{O}_{4}$

\begin{tabular}{cccc}
\hline Amostra & $\mathbf{M}_{\mathbf{S}}(\mathbf{e m u} / \mathbf{g})$ & $\mathbf{M}_{\mathbf{R}}(\mathbf{e m u} / \mathbf{g})$ & $\mathbf{H}_{\mathbf{C}}(\mathbf{G})$ \\
\hline $\mathrm{ZnFe}_{2} \mathrm{O}_{4}$ & 47,154 & 0,83560 & 33,627 \\
$\mathrm{Ni}_{0,25} \mathrm{Zn}_{0,75} \mathrm{Fe}_{2} \mathrm{O}_{4}$ & 70,726 & 1,8368 & 41,612 \\
$\mathrm{Ni}_{0,50} \mathrm{Zn}_{0,50} \mathrm{Fe}_{2} \mathrm{O}_{4}$ & 119,53 & 1,6918 & 17,401 \\
$\mathrm{Ni}_{0,75} \mathrm{Zn}_{0,25} \mathrm{Fe}_{2} \mathrm{O}_{4}$ & 48,148 & 2,0835 & 42,968 \\
$\mathrm{NiFe}_{2} \mathrm{O}_{4}$ & $\mathrm{NS}$ & 0,33382 & 135,36 \\
\hline
\end{tabular}

NS- amostra não apresentou saturação 
A Figura 4 mostra a dependência resistividade elétrica em corrente contínua pela temperatura para todas as amostras de ferritas. Em todas as amostras, verificou-se que a resistividade do material diminui em função da temperatura, como ocorre nos semicondutores.

A Figura 5 apresenta-se o gráfico do $\ln (\rho \mathrm{T}-1 / 2)$ vs $1 / \mathrm{T}$ para as ferritas NZFO A partir de uma análise mais detalhada é possível indicar uma grande região linear associada com o modelo de barreira /cristalito. O limite de cristalitos com modelo de barreiras potenciais é o mecanismo de condução elétrica observado para todas as amostras em altas temperaturas. Para eles, o alto valor da resistividade está associado à presença simultânea de íons ferrosos e férricos em sítios de rede equivalentes.

O gráfico de Arrhenius mostra que o aumento da temperatura diminui a resistividade e, portanto, a condutividade aumenta. Isso está de acordo com o fato de que a ferrita, sob investigação, tem comportamento semicondutor. $\mathrm{O}$ decréscimo na resistividade pode ser explicado sob a forma da presença de íons $\mathrm{Fe}^{+2}$ à medida que o zinco é adicionado, que são produzidos durante a sinterização [20]. As ferritas de espinélio se comportam como um semicondutor com baixa mobilidade de portadores de carga e dependência exponencial da condutividade elétrica com a temperatura. No entanto, o mecanismo de condução dos portadores de carga é bastante diferente do apresentado pelo semicondutor [21]. A diminuição pode ser explicada sob a forma da presença de Íons $\mathrm{Fe}^{+2}$ à medida que o $\mathrm{Zn}$ é adicionado, que são produzidos durante sinterização.

Por meio do espectro de impedância é possível determinar a permissividade. As pastilhas cilíndricas são tratadas como capacitores de placas paralelas. Sejam os parâmetros da condução elétrica em corrente alternada: $A_{S}$ como área superficial da pastilha, $\mathrm{Cp}$ a capacitância e $\epsilon$ a permissividade. A Figura 6 apresenta o gráfico Nyquist para as ferritas Níquel- Zinco. O eixo das abscissas dos diagramas de Nyquist representa a parte real da impedância e o eixo das ordenadas, a parte imaginária, ambos em $\mathrm{M} \Omega$. A Tabela 2 apresenta os parâmetros da condução elétrica.

Asamostras $\mathrm{Ni}_{0.50} \mathrm{Zn}_{0,50} \mathrm{Fe}_{2} \mathrm{O}_{4}$ eNi $\mathrm{Ni}_{0,75} \mathrm{Zn}_{0.25} \mathrm{Fe}_{2} \mathrm{O}_{4}$ apresentaram dois arcos de impedância referente ao interior do grão e ao contorno de grão. O primeiro corresponde à altas frequências, associado às propriedades condutoras do interior do grão.

Tabela 2. Parâmetros da condução elétrica em corrente alternada das ferritas NZFO

\begin{tabular}{cccc}
\hline Amostra & $\mathbf{A}_{\mathbf{s}} \mathbf{1 0}^{-4}\left(\mathbf{m}^{\mathbf{2}}\right)$ & $\mathbf{C}_{\mathbf{p}} \mathbf{1 0}^{-\mathbf{1 1}}(\mathbf{F})$ & $\boldsymbol{\varepsilon}$ \\
\hline NZF0 & 0,622 & 6,50 & 224 \\
NZF025 & 0,622 & 0,158 & 544 \\
NZF050 & 0,622 & 5,32 & 183 \\
NZF075 & 0,622 & 9,84 & 339 \\
NZF1 & 0,622 & 1,50 & 51,7 \\
NZF050(2) & 0,622 & 9,79 & 338 \\
NZF075(2) & 0,622 & 0,699 & 2410 \\
\hline
\end{tabular}

* (2) dados referentes a impedância no interior do grão das amostras NZF50 e NZF75.

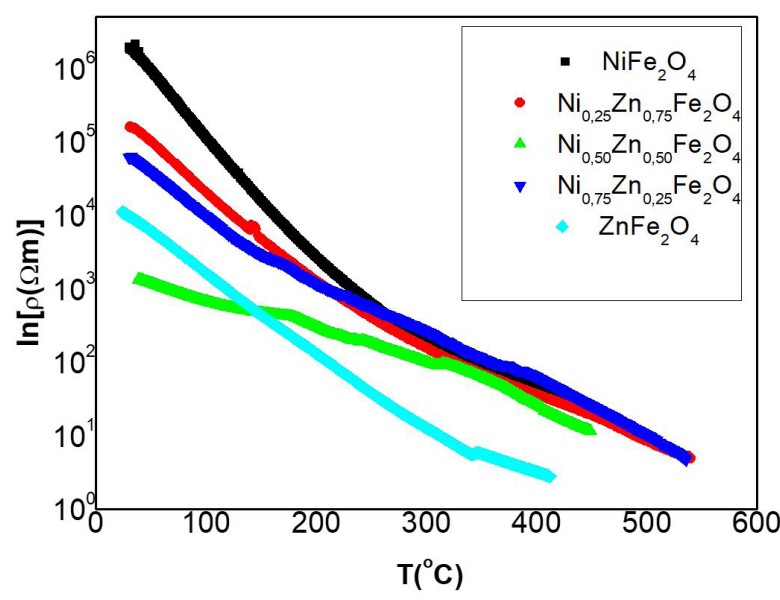

Figura 4. Resistividade elétrica da ferrita níquel- zinco.

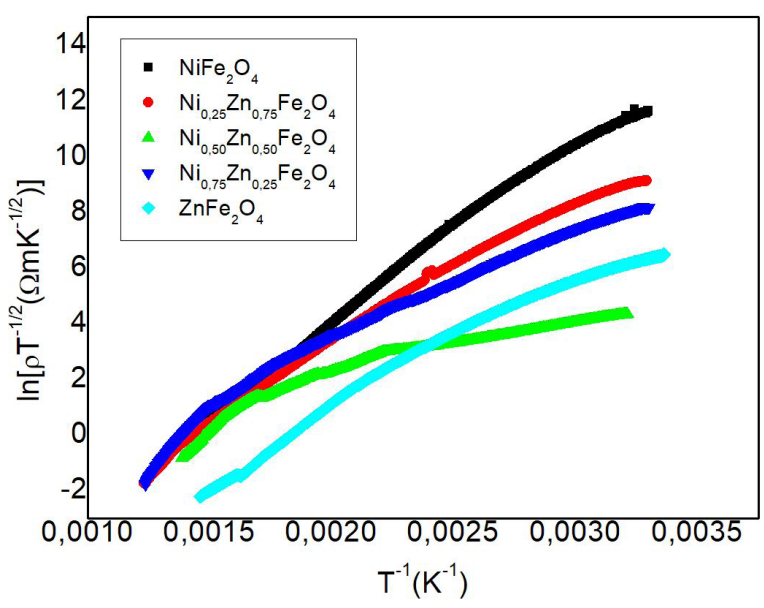

Figura 5. Dependência da resistividade elétrica em relação à temperatura através da relação $\ln \left(\rho \mathrm{T}^{-1 / 2}\right)$ vs. 1/T para as ferritas níquel- zinco.

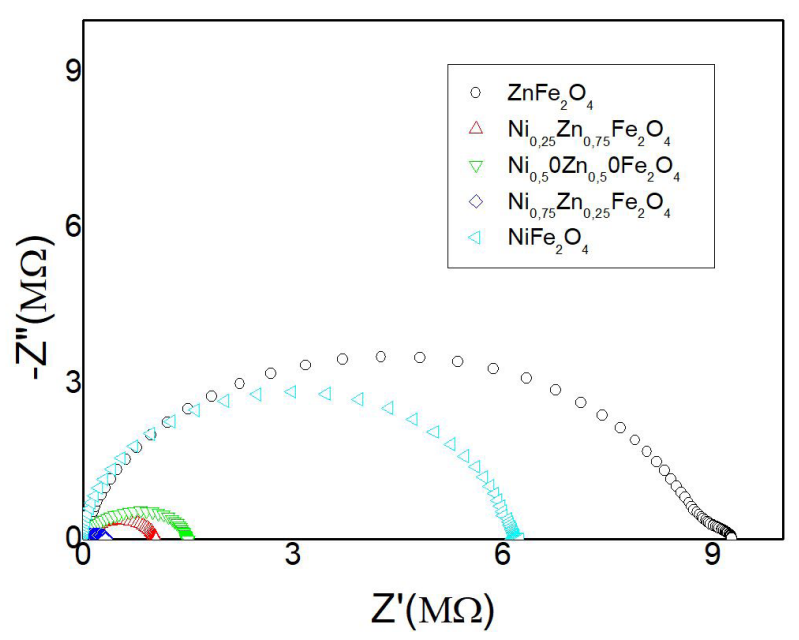

Figura 6. Espectro de impedâncias para as ferritas níquel- zinco. 
Tabela 3. Parâmetros do modelo de condução elétrica por barreira para as $\mathrm{Ni}_{\mathrm{x}} \mathrm{Zn}_{1-\mathrm{x}} \mathrm{Fe}_{2} \mathrm{O}_{4}$

\begin{tabular}{ccccc}
\hline Amostra & $\mathbf{L} / \mathbf{2}\left(\mathbf{1 0}^{-\mathbf{m}} \mathbf{)}\right.$ & $\mathbf{N}_{\mathbf{d}}\left(\mathbf{1 0}^{\mathbf{4 2}} \mathbf{m}^{-\mathbf{3}}\right)$ & $\mathbf{L}_{\mathbf{D}}\left(\mathbf{1 0}^{\mathbf{- 1}} \mathbf{m}\right)$ & $\mathbf{\mathbf { E } _ { \mathbf { b } }}(\mathbf{e V})$ \\
\hline NZF0 & 4,05 & 3,77 & $2,060 \mathrm{E} 10^{-10}$ & 0,638 \\
NZF025 & 4,33 & 8,00 & 8,704 & 0,509 \\
NZF050 & 3,96 & 3,23 & 7,958 & 0,510 \\
NZF075 & 4,04 & 5,73 & 8,122 & 0,334 \\
NZF1 & 4,12 & 0,839 & 8,285 & 0,375 \\
NZF050(2) & 3,96 & 5,95 & 7,958 & 0,510 \\
NZF075(2) & 4,04 & 40,8 & 8,122 & 0,334 \\
\hline
\end{tabular}

As frequências intermediárias têm um segundo semicírculo, sendo relacionado com os contornos de grão.

Os valores de energia de barreira $\mathrm{E}_{\mathrm{B}}$ são obtidos pela inclinação da linha do gráfico $\ln \left(\rho \mathrm{T}^{-1 / 2}\right)$ versus $1 / \mathrm{T}$. A concentração de portadores $\mathrm{Nd}$ e o comprimento de Debye LD são determinados pelas Equações 1 e 2, respectivamente. Outro parâmetro que compõe este modelo de condução é a permissividade é que determinada pelo espectro de impedância. O comprimento médio do cristalito, $L / 2$, é determinado pela equação de Scherrer através da técnica de difração de raios X. A Tabela 3 apresenta os parâmetros característicos para o modelo de condução elétrica por barreira.

Conforme determinado pelos parâmetros de ajuste na Tabela 2, a condutividade elétrica de todas as amostras de ferrita níquel e zinco depende dos contornos. Os valores dessas barreiras são altos o suficiente para evitar que cargas transportadoras atravessem os limites do cristalito, e o baixo o valor $\mathrm{L}_{\mathrm{D}}$ confirma este fato.

Portanto, podemos concluir que o transporte elétrico nas amostras ferritas policristalinas estudadas são dominadas por esses efeitos de contorno. As barreiras de energia de ordem $0,5 \mathrm{e} \mathrm{V}$ caracterizam as amostras de $\mathrm{Ni}_{\mathrm{x}} \mathrm{Zn}_{1-\mathrm{x}} \mathrm{Fe}_{2} \mathrm{O}_{4}$ como ferritas semicondutoras. As energias de ativação relacionam com a resistividade com um fator exponencial $\mathrm{T}^{1 / 2}$, característica de condução limitada por barreiras. Esse modelo é assegurado pela relação do comprimento de Debye menor que o tamanho médio do cristalito $\left(\mathrm{L}_{\mathrm{D}}<\mathrm{L} / 2\right)$.

\section{Conclusão}

$\mathrm{O}$ sistema $\mathrm{Ni}_{\mathrm{x}} \mathrm{Zn}_{1-\mathrm{x}} \mathrm{Fe}_{2} \mathrm{O}_{4}$ é uma ferrita que apresenta estrutura cristalina tipo espinélio para todas as composições. As curvas de magnetização versus campo magnético coletadas são características de materiais magnéticos moles. A amostra $\mathrm{Ni}_{0,75} \mathrm{Zn}_{0,25} \mathrm{Fe}_{2} \mathrm{O}_{4}$ apresentou maiores valores de campo coercitivo e magnetização remanescente. Na caracterização elétrica verificou-se que as ferritas apresentam um comportamento semicondutor e possuem um alto valor de resistência a temperatura ambiente. À altas temperaturas verificaram-se que o mecanismo de condução nas ferritas se dá por barreiras de potencial localizados nos limites do cristalito se dá em uma região $(T=240 \mathrm{~K})$, pois a condição de $\mathrm{L}_{\mathrm{D}}<\mathrm{L} / 2$ foi satisfeita.
Tais resultados mostram que as ferritas de níquel-zinco apresentam características interessantes para o desenvolvimento de novos dispositivos eletrônicos. Além disso, também podem ser aplicadas em dispositivos magnéticos, pois possuem valores altos de magnetização de saturação.

\section{Agradecimentos}

Os autores agradecem às agências brasileiras, Conselho Nacional de Desenvolvimento Científico e Tecnológico (CNPq), Coordenação de Aperfeiçoamento de Pessoal de Nível Superior (CAPES), Fundação de Amparo à Pesquisa do Estado de Minas Gerais (FAPEMIG) e Instituto Nacional de Metrologia, Qualidade e Tecnologia (Inmetro- especialmente Dimci/ Diele /Lamat).

\section{Referências}

1 Schmalzried H. Chemical kinetics of solids. Verlag Chemie: Weinheim; 1995.

2 Oliveira VD, Rubinger M, Silva MR, Oliveira AF, Rodrigues G, Ribeiro VAS. Magnetic and electrical properties of $\mathrm{MnxCu} 1-\mathrm{xFe} 2 \mathrm{O} 4$ ferrite. Materials Research. 2016;19(4):786-790.

3 Baruwati B, Guin D, Manorama SV. Pd on surface modified $\mathrm{NiFe} 2 \mathrm{O} 4$ nanoparticles: a magnetically recoverable catalyst for suzuki and heck reactions. Organic Letters. 2007;9(26):5377-5380.

4 Shultz MD, Calvin S, Fatouros PP, Morrison SA, Carpenter EE. Enhanced ferrite nanoparticles as MRI contrast agents. Journal of Magnetism and Magnetic Materials. 2007;311(1):464-468.

5 Karakaş ZK, Boncukcuoğlu R, Karakaş IH, Ertuğrulc $M$. The effects of heat treatment on the synthesis of nickel ferrite (nife2o4) nanoparticles using the microwave assisted combustion method. Journal of Magnetism and Magnetic Materials. 2015;374:298306.

6 Sato T, Masai A, Ota Y, Sato H, Matuski H, Yanada $\mathrm{T}$, et al. The development of anticancer agent releasing microcapsule made of ferromagnetic 
amorphous flakes for intratissue hyperthermia. IEEE Transactions on Magnetics. 1993;29:3325-3330.

7 Mansour SF. Frequency and composition dependence on the dielectric properties for $\mathrm{Mg}-\mathrm{Zn}$ ferrite. Egyptian Journal of Solids. 2005;28(2):263-273.

$8 \mathrm{Pu} \mathrm{S}$, Liu M. Tunable photonic crystals based on $\mathrm{MnFe}_{2} \mathrm{O}_{4}$ magnetic fluids by magnetic fields. Journal of Alloys and Compounds. 2009;481:851-854.

9 Hirosawa F, Iwasaki T, Watano S. Synthesis and magnetic induction heating properties of Gd-substituted Mg-Zn ferrite nanoparticles. Applied Nanoscience. 2017;7:209-214.

10 Zhang S, Lee W. Spinel-containing refractories., In: Schacht CA, editor. Refractories handbook. New York: Marcel Dekker; 2004. p. 215.

11 Carta D, Casula MF, Falqui A, Lo Che D, Mountjoy $\mathrm{G}$, Sangregorio $\mathrm{C}$, et al. A structural and magnetic investigation of the inversion degree in ferrite nanocrystals $\mathrm{MFe}_{2} \mathrm{O}_{4}(\mathrm{M}=\mathrm{Mn}, \mathrm{Co}, \mathrm{Ni})$. The Journal of Physical Chemistry C. 2009;113:8606-8615.

12 IHsiang H, Wu JL. Copper-rich phase segregation effects on the magnetic properties and DC-biassuperposition characteristic of $\mathrm{NiCuZn}$ ferrites. Journal of Magnetism and Magnetic Materials. 2015;374:367-371.

13 Goldman A. Modern ferrite technology. New York: Springer; 2006.

14 Yahya N, Aripin ASMN, Aziz AA, Daud H, Zaid HM, Pah LK, et al. Synthesis and charaterization of magnesium zinc ferrites as electromagnetic source. American Journal of Engineering and Applied Sciences. 2008;1:53-56.
15 Gabal MA, El-Shishtawy RM, Al Angari YM. Structural and magnetic properties of nano-crystalline ni-zn ferrites synthesized using egg-white precursor. Journal of Magnetism and Magnetic Materials. 2012;324(14):2258-2264.

16 Mancini MW, Paulin Filho PI. Barreiras de potencial mapeadas por microscopia de força atômica em cerâmicas à base de $\mathrm{BaTiO} 3$ dopadas com lantânio. Cerâmica. 2007;53:147-152.

17 Montoya ACA. Produção de filmes de Dióxido de titânio para aplicações fotovoltaicas [dissertação] Itajubá: Universidade Federal de Itajubá; 2014.

18 Mardare D, Iftimie N, Crişan M, Răileanu M, Yildiz A, Coman T, et al. Electrical conduction mechanism and gas sensing properties of Pd-doped TiO2 films. Journal of Non-Crystalline Solids. 2011;357(7):17741779 .

19 Parmar H, Upadhyay RV, Rayaprol S, Siruguri V. Structural and magnetic properties of nickel-zinc ferrite nanocrystalline magnetic particles prepared by microwave combustion method. Indian Journal of Physics. 2014;88:1257-1264.

20 Islam MU, Abbas T, Niazi SB, Ahmed Z, Sabeen S, Chaudhry MA. Electrical behavior of fine particle, Co-precipitation prepared Ni-Zn ferrites. Solid State Communications. 2004;130(5):353-356.

21 Murthy VRK, Sundaram S, Viswanathan B. Microwave materials. Berlim: Springer- Verlag; 1994.

Recebido em: 10 Out. 2019

Aceito em: 21 Jul. 2021 